\title{
Optical Metrology with many faces
}

\author{
Malgorzata Kujawinska, ${ }^{*}$ \\ ${ }^{1}$ Institute of Micromechanics and Photonics, Warsaw University of Technology, Sw. A. Boboli 8, 02-525 Warszawa,
}

Received June 29, 2012; accepted June 30, 2012; published June 30, 2012

\begin{abstract}
A brief editorial overview of the current issue is presented. This special issue of Photonics Letters of Poland devoted to optical metrology contains 15 invited and regularly submitted letters showing a wide range of optical measurement and characterization methods, data processing procedures and their applications.
\end{abstract}

Most of the technical and biomedical activities require measurements of different quantities. Optics and photonics provide an excellent tool to capture data in the form of a stream of point wise intensity values or images (full-field methods) which can be converted into digital information on required physical quantities. Optical metrology since long time has been important and continuously growing field of science and engineering. This fact is well illustrated in the special issue of Photonics Letters of Poland which I have the pleasure to present to the readers.

The first two papers are devoted to new applications and configurations of digital holography. Kemper with colleagues presents measurements of changes of the volume and the intracellular solute concentrations of biological cells by means digital holography microscope. Kujawinska et al. describe wide angle digital holographic system with the capability of real-time optical reconstruction.

Next paper refers to noncoherent methods, specifically, Maczkowski et al. propose an optical measurement system which uses structure light projection, multispectral color acquisition and Bi-Directional Reflectance Distribution Function estimation for multimodal digitization of cultural heritage objects.

Yamaguchi discuss the conditions for application of digital speckle correlation method for displacement measurements of curved surfaces.

Tomczewski and his colleagues present new applications of low coherence interferometry and optical coherence tomography with non-Gaussian light source.

Next three papers are devoted to new methods and algorithms of fringe pattern analysis and enhancement. Wegierow and others propose a new multispectral phase shifting interferometry algorithm. Sunderland et al. present the method of deriving shape and optical thickness

${ }^{*}$ E-mail: m.kujawinska@mchtr.pw.edu.pl variations of a plate which requires recording two interferograms only. Wielgus and Patorski discuss the notion of non-local filtration in the context of fringe pattern images processing.

Ottevaere with colleagues present a micro-optical system for UV/VIS absorbance detection in silicon microfluidic channels and demonstrate the proof-of-concept of this microfluidic light probe by measuring standard samples of coumarin dye.

The next two papers are devoted to numerical analysis of photonics crystal fibres (Badhdasaryan et al.) and both theoretical and experimental studies of 1-D photonics crystals for photovoltaic (Karasiński, Gondek). This together with the next two papers focused on technology of special films (Maheswari et al.) and liquid crystal blue phase interesting for fast light modulators and tunable photonic crystals (Chojnowska et al.) are providing the examples of recent challenges for optical metrology.

In the last paper Schmit and Reed come back to the methods for measurement of the mechanical and structural properties of biological samples in nanoscale.

Of course the papers in this special issue do not cover all activities and recent trends in optical metrology, but they are good examples of this exciting field of optics and photonics ranging in applications from engineering and biological structures to multimedia. 